\title{
Study of Air Cooling System for Temperature Controlled Steel Rods
}

\author{
Jun-Tae Lee, Seungjin Lee, Seongkyu See \\ POSCO \\ Goedong-dong Nam-gu, Pohang Gyeongbuk, Korea \\ joontae@posco.com; bloodone@posco.com; seessk@posco.com
}

\section{Extended Abstract}

The purpose of this study is to analyze cooling mechanism in hot rolled wire rods. Wire rod air cooling system has been popularly applied in a number of mills since the air cooling system has many advantages such as high production rate and low operation cost [1]. However, there is expected temperature difference between edge and center part in hot rolled wire rods caused by the mass density difference and cooling rate. Temperature difference is one of the most important reasons to cause mechanical property changes in wire rods.

A numerous studies have been developed to overcome these problems. Mist cooling process is applied to reduce the temperature difference [2]. To solve the problems of limitation of cooling medium, mist is sprayed directly or indirectly to the edge of a coil, making higher cooling rate on the edge part.

This study researched air cooling mechanism to produce steel rods with no mechanical property changes. Numerical simulations have been performed to evaluate the cooling process in wire rod air cooling system. The result of numerical simulation can be used to design novel air cooling system; temperature controlled air cooling system. Using electronic heaters, cooling rate can be controlled in center part of the rolled wire rods. The novel air cooling system can be a more effective tool to overcome the problems in steel rods cooling method and the findings may be useful to achieve uniform temperature distribution of wire rods with slow cooling rate.

\section{References}

[1] S. E. Lundberg, "A vision of wire rod rolling technology for the twenty first century," Advanced Materials Research, vol. 23, pp. 39-44, 2007.

[2] R. D. Morales, A. G. Lopez and I. M. Olivares, "Heat transfer analysis during water spray cooling of steel rods," ISIJ International, vol. 30, no. 1, pp. 48-57, 1990. 